\title{
Escala de Sobrequalificação Percebida: Adaptação e evidências de validade
}

João Moreira Gonçalves Neto. Universidade de Brasília.

Jairo Eduardo Borges-Andrade. Universidade de Brasília.

\section{Resumo}

Sobrequalificação percebida é a situação na qual o indivíduo se percebe possuidor de qualificação maior do que aquela exigida por seu trabalho. Tal situação tem sido encontrada relacionada a atitudes negativas e comportamentos indesejados no trabalho. No Brasil, não há instrumentos para mensuração da sobrequalificação percebida. O objetivo deste estudo foi adaptar uma escala de sobrequalificação percebida e analisar suas evidências de validade. A escala foi traduzida e retrotraduzida, e aplicada a 222 trabalhadores. Análise fatorial exploratória encontrou uma solução hierárquica (dois fatores de primeira ordem e um de segunda ordem). Análise fatorial confirmatória, em amostra de 265 trabalhadores, deu suporte à solução hierárquica, embora com ajustamento pobre. A versão brasileira apresentou evidências de validade e confiabilidade para mensuração da sobrequalificação percebida. Sugeriram-se novos estudos para testar a estrutura fatorial em outras amostras.

Palavras-chaves: psicometria; análise fatorial; medidas de atitude; atitudes do empregado; comportamento organizacional.

\begin{abstract}
Scale of Perceived Overqualification: Adaptation and Validity Evidences. Perceived overqualification is the situation in which an individual perceives that they have qualification higher than that required by their job. Such situation has been found related to negative attitudes and undesirable behaviors at work context. In Brazil, there are no instruments for measuring perceived overqualification. This study aimed to adapt a scale of perceived overqualification and to analyze for its validity evidences of. The scale was translated and back-translated, and applied to 222 workers. Exploratory factor analysis found a hierarchical solution (two first-order factors and one second-order factor). Confirmatory factor analysis, in a sample of 265 workers, supported the hierarchical solution, although with poor fit. The Brazilian version showed validity and reliability evidences for measuring perceived overqualification. Other studies were suggested in order to test the factor structure in other samples.
\end{abstract}

Keywords: psychometrics; factor analysis; attitude measures; employee attitudes; organizational behavior.

\section{Resumen}

Escala de Sobrecualificación Percibida: Adaptación y Evidencias de Validez. Sobrecualificación percibida es la situación en la que un individuo percibe que tiene calificación más grande de la que se exige en su trabajo. Esta situación ha sido encontrada relacionada con actitudes negativas y comportamientos indeseables en el contexto del trabajo. En Brasil, no hay herramientas de valoración de la sobrecualificación percibida. El objetivo de esta investigación fue una adaptación de una escala de sobrecualificación percibida y un análisis de sus evidencias de validez. La escala fue traducida y retrotraducida, y se aplicó a 222 trabajadores. El análisis factorial exploratorio encontró una estructura jerárquica (dos factores de primer orden y un de segundo orden). El análisis factorial confirmatorio, con muestra de 265 trabajadores, apoyó la estructura jerárquica, aunque con el ajuste deficiente. La versión brasileña presentó evidencias de validez y fiabilidad de valoración de la sobrecualificación percibida. Se propusieron nuevas investigaciones para probar la estructura factorial en otras muestras.

Palabras clave: psicometría; análisis factorial; medidas de actitud; actitud del empleado; comportamiento organizacional. 
O nível de qualificação dos trabalhadores pode constituir um problema para as organizações. Quando esse nível é baixo, as organizações podem ter custos com treinamento, a fim de alcançar índices adequados de desempenho e produtividade. Entretanto, o alto nível de qualificação, conhecido como sobrequalificação, também pode representar um problema e tem despertado o interesse dos pesquisadores (Deng et al., 2018; Lobene \& Meade, 2013).

Sobrequalificação pode ser definida como a condição na qual um indivíduo possui qualificação, compreendida como educação formal, habilidades e experiência profissional, maior do que a exigida por seu trabalho (Maynard, Joseph, \& Maynard, 2006). Ela tem sido relacionada a atitudes negativas (como baixo comprometimento com a organização e insatisfação no trabalho) e a comportamentos indesejados (como comportamentos contra-producentes e rotatividade) no trabalho (Maynard, Brondolo, Connelly, \& Sauer, 2015).

A definição constitutiva do constructo sobrequalificação, conforme modelo adotado neste estudo, está fundamentada em teorias de subemprego (Feldman, 1996), congruência pessoa-trabalho (Edwards, 1991; Kristof, 1996) e sobrequalificação (Maynard et al., 2006). Subemprego é definido como a discrepância entre o indivíduo e seu cargo ou trabalho com base em algum critério (Feldman, 1996), tendo sido propostas cinco dimensões: mais educação formal do que a exigida pelo cargo; mais habilidades e experiência de trabalho do que as exigidas pelo cargo; aceitação involuntária de emprego em área diferente daquela para a qual recebeu formação; aceitação involuntária de emprego temporário, de meio-período ou intermitente; e remuneração menor do que aquela recebida em empregos anteriores ou menor do que a de outros indivíduos com mesma formação.

A congruência pessoa-trabalho consistiria na comparação entre características do indivíduo e características do cargo ou trabalho, considerando-se os recursos e necessidades tanto do indivíduo quanto da organização (Edwards, 1991; Kristof, 1996). Essa congruência seria composta por duas díades: necessidades-suprimentos, correspondendo à compatibilidade entre as necessidades do indivíduo (necessidades psicológicas, valores, preferências, etc.) e os recursos disponibilizados pela organização (como remuneração e participação nas decisões); e demandas-aptidões, correspondendo à compatibilidade entre as demandas do trabalho (como carga de trabalho) e os recursos do indivíduo (como experiência e educação formal).

A fim de definir o constructo sobrequalificação, Maynard et al. (2006) utilizaram as teorias de subemprego (Feldman, 1996) e de congruência pessoa-trabalho (Edwards, 1991; Kristof, 1996). Consideraram a sobrequalificação como correspondente a duas das dimensões do subemprego: mais educação formal do que a exigida pelo cargo; e mais habilidades e experiência de trabalho do que as exigidas pelo cargo. A sobrequalificação seria então um caso de incongruência pessoa-trabalho; especificamente, incongruência demandas-aptidões, isto é, o indivíduo possuiria mais recursos (educação formal, habilidades e experiência) do que os demandados por seu trabalho (Maynard et al., 2006).

Sendo a sobrequalificação percebida um constructo, para ser mensurável ela deveria ser o atributo de algum objeto (Pasquali, 2010). Se a sobrequalificação percebida corresponde a duas das dimensões do subemprego (Maynard et al., 2006), então, ela poderia ser considerada um atributo do subemprego, o qual seria seu objeto. Se a sobrequalificação percebida corresponde à incongruência demandas-aptidões e as aptidões correspondem à educação formal, experiência profissional e habilidades, então, estas seriam dimensões da sobrequalificação percebida. Portanto, no modelo da sobrequalificação, adotado neste estudo, a sobrequalificação percebida seria um atributo do subemprego (seu objeto) e teria educação formal, experiência profissional e habilidades como suas dimensões.

Dois tipos principais de sobrequalificação têm sido propostos: objetiva e percebida. A sobrequalificação objetiva é observada externamente comparando-se, por exemplo, o nível de educação formal que um indivíduo possui ao nível de educação formal exigido pelo cargo que ocupa. A sobrequalificação percebida é observada subjetivamente pelo próprio indivíduo, ao avaliar o quanto se considera qualificado para seu trabalho. Essa última tem sido a mais estudada, sob a justificativa de que a percepção do indivíduo acerca da relação entre sua qualificação e seu trabalho seria mais relevante para seu comportamento, do que a relação real qualificação-trabalho (Hu et al., 2015; Johnson, Morrow, \& Johnson, 2002).

Apesar de independentes, as sobrequalificações percebida e objetiva estariam inter-relacionadas (Maltarich, Reilly, \& Nyberg, 2011). Por exemplo, a sobrequalificação objetiva (mensurada pela 
sobre-educação) foi encontrada moderadora da relação entre merecimento narcisista e sobrequalificação percebida. A relação encontrada foi positiva em sua presença e não significativa em sua ausência (Maynard et al., 2015). A sobrequalificação percebida foi ainda encontrada maior para um grupo de indivíduos objetivamente sobrequalificados (novamente mensurada por meio da sobre-educação) do que para um grupo de indivíduos compatíveis com o cargo (Zhang, Law, \& Lin, 2016).

Estudos acerca das consequências da sobrequalificação percebida encontraram-na positivamente relacionada a: raiva quanto à situação de emprego (Liu, Luksyte, Zhou, Shi, \& Wang, 2015); depressão e estresse (Wassermann \& Hoppe, 2019); sintomas somáticos (Triana, Trzebiatowski, \& Byun, 2017); cyberloafing (Cheng, Zhou, Guo, \& Yang, 2018), comportamentos contraproducentes (Liu et al., 2015) e de afastamento do trabalho (Triana et al., 2017). A sobrequalificação percebida foi ainda encontrada relacionada negativamente a: bem-estar subjetivo (Erdogan, Tomás, Valls, \& Gracia, 2018); satisfação com a vida (Wassermann \& Hoppe, 2019); satisfação no trabalho (Alfes, Shantz, \& Baalen, 2016; Wassermann, Fujishiro, \& Hoppe, 2017); auto-estima baseada na organização (Liu et al., 2015); e comportamento adaptativo no trabalho (Wu, Tian, Luksyte, \& Spitzmueller, 2017).

Para mensuração da sobrequalificação percebida, um dos principais instrumentos é a Scale of Perceived Overqualification (SPOQ), desenvolvida por Maynard et al. (2006). A SPOQ tem sido utilizada em diversos estudos recentes, que ajudaram a estabelecer as bases sobre o que se sabe a respeito da sobrequalificação percebida, como Alfes et al. (2016), Cheng et al. (2018), Deng et al. (2018), Erdogan et al. (2018), Jackson e Collings (2018), Liu et al. (2015), Maynard et al. (2015), Tebbe, Allan e Bell (2019), Wassermann et al. (2017), Wassermann e Hoppe (2019) e Zhang et al. (2016).

Três estudos foram conduzidos para o desenvolvimento da SPOQ (Maynard et al., 2006). No primeiro, a análise de componentes principais (ACP) resultou em um componente, composto por nove itens, com cargas entre 0,72 e 0,80 e consistência interna (alfa de Cronbach) de 0,89, explicando $58 \%$ da variância. O segundo analisou a precisão da escala, via delineamento teste-reteste, e encontrou um alfa de 0,92. No terceiro estudo, que testou a relação entre sobrequalificação e atitudes no trabalho, o escore da SPOQ se correlacionou negativamente ao comprometimento afetivo e às satisfações com salário, promoções, colegas, supervisão e trabalho, e se correlacionou positivamente à intenção de saída.

No Brasil, poucos estudos publicados abordaram a sobrequalificação percebida, entre eles, Maciel e Camargo (2013) e Weymer, Maciel e Castor (2014). Para mensuração da sobrequalificação percebida, esses autores utilizaram traduções livres de itens do instrumento de Johnson et al. (2002). Isso porque não há instrumentos brasileiros para a mensuração da sobrequalificação percebida, uma lacuna que dificulta seu estudo em amostras nacionais.

Os valores associados ao trabalho, à educação e à qualificação profissional podem variar entre culturas e provavelmente estão associados às crenças dos indivíduos acerca da sobrequalificação. Tais crenças, por sua vez, podem estar associadas à percepção que o indivíduo desenvolve sobre esse constructo no seu contexto de trabalho. É essencial dispor de uma medida apropriada no Brasil, como primeiro passo para fundamentar uma linha de pesquisa que investigue seus efeitos em variáveis de micro comportamento organizacional.

A elaboração de instrumentos para mensuração de constructos psicológicos pode seguir dois caminhos: desenvolver um instrumento ou adaptar um já existente em outro idioma. A segunda opção apresenta, como vantagens, permitir a comparação de resultados obtidos em amostras de contextos ou populações distintos e oferecer maior possibilidade de generalização de resultados (Borsa, Damásio, \& Bandeira, 2012). Considerando as razões anteriormente apresentadas, o objetivo desta pesquisa foi adaptar e analisar evidências de validade e confiabilidade da SPOQ, por meio de dois estudos. A SPOQ foi escolhida para adaptação por ser a mais ajustada ao modelo da sobrequalificação adotado nesta pesquisa.

\section{Estudo 1}

O Estudo 1 teve os objetivos de (1) adaptar os itens da SPOQ ao português brasileiro, a fim de constituírem a Escala de Sobrequalificação Percebida (ESQP) e (2) explorar sua estrutura fatorial.

\section{Método}

\section{Participantes}

Obteve-se amostra de 222 trabalhadores (58\% homens), com média de idade de 33,98 anos ( $D P=$ 9,49 ), oriundos das cinco regiões brasileiras (norte: $39 \%$; 
nordeste: $4 \%$; centro-oeste: $14 \%$; sudeste: $35 \%$; sul: $8 \%)$. Quanto ao nível de educação formal: $1 \%$ possuíam ensino fundamental; $24 \%$, ensino médio; $28 \%$, graduação; e 47\%, pós-graduação. Trabalhavam nos setores público (45\%) e privado (55\%), em média, havia 6,19 anos ( $D P=6,71)$, ocupando cargos que exigiam nível fundamental (4\%), médio (24\%), superior (48\%) e pós-graduação (18\%). Para os cargos de $6 \%$ dos participantes, não havia exigência de escolaridade mínima ou não souberam responder.

\section{Instrumentos}

Os participantes responderam à versão da SPOQ adaptada ao português brasileiro. A SPOQ consiste em um instrumento unidimensional, composto por nove itens respondidos em escala de sete pontos ( 1 = discordo totalmente; 7 = concordo totalmente). Trata-se de um instrumento para mensuração direta da sobrequalificação (Kristof, 1996), isto é, fornece uma medida direta da incongruência percebida entre a qualificação exigida pelo cargo e a possuída pelo indivíduo. Os participantes responderam ainda a um questionário sociodemográfico.

\section{Procedimentos de Adaptação}

A definição operacional do constructo foi realizada por meio de processo de tradução e retrotradução. Assumiu-se que os itens do instrumento a ser adaptado eram, em seu idioma original, representantes legítimos do constructo investigado. Assim, ao adaptá-los ao novo idioma, o português brasileiro, havendo equivalência de conteúdo entre os itens do instrumento original e da versão brasileira, essa legitimidade representativa seria mantida no novo instrumento. Esse constitui o foco do processo de tradução e adaptação (Beaton, Bombardier, Guillemin, \& Ferraz, 2000).

O processo de tradução e retrotradução ocorreu em quatro fases: tradução do idioma de origem (inglês) para o idioma-alvo (português brasileiro), síntese das traduções, retrotradução do idioma-alvo para o idioma de origem e síntese das retrotraduções (Beaton et al., 2000; Borsa et al., 2012). Algumas fases descritas por Beaton et al. (2000) e Borsa et al. (2012), como as análises de juízes (especialistas e público alvo), foram consideradas não aplicáveis ao presente estudo, devido à baixa complexidade do instrumento sob adaptação.

$\mathrm{Na}$ fase de tradução, a SPOQ foi encaminhada a três tradutores, cujos idiomas maternos eram o idioma-alvo e possuíam fluência no idioma de origem, a fim de assegurar a acurácia da tradução quanto às especificidades do idioma-alvo (Beaton et al., 2000). Esses tradutores não pertenciam à área da psicologia, sendo leigos quanto à teoria do constructo abordado. Cada um deles elaborou, independentemente, uma tradução da escala (T1, T2 e T3). Na fase de síntese das traduções, dois pesquisadores analisaram as três traduções, em busca de possíveis divergências de sentido e elaboraram uma única versão traduzida (T123).

$\mathrm{Na}$ fase de retrotradução, a versão T123 foi enviada a três outros tradutores. Esses eram nativos no idioma de origem, fluentes no idioma-alvo e também desconheciam a SPOQ. Cada tradutor elaborou uma tradução independente (R1, R2 e R3) da versão T123, do português brasileiro para o inglês. Em seguida, na fase de síntese das retrotraduções, as versões R1, R2 e R3 foram analisadas por um quarto tradutor (nativo no idioma de origem e fluente no idioma-alvo), que elaborou uma versão retrotraduzida final, R123.

A fim de analisar a equivalência semântica entre os itens das versões original e retrotraduzida, a versão R123 foi encaminhada a um dos autores da SPOQ, o qual apontou possíveis problemas nos Itens 1 (o termo qualification) e 4 (o termo training). Esses itens foram alterados, em ambas as versões R123 (substituição dos termos training e qualification por formal education) e T123 (substituição do termo formação por educação formal). Uma vez assegurada a equivalência semântica entre as versões original e R123, inferiu-se que tal equivalência também foi garantida entre a versão original e a versão T123, a partir de então denominada ESQP. A escala de respostas da ESQP foi mantida semelhante a da SPOQ, utilizando escala de sete pontos.

\section{Procedimentos de Coleta e Análise de Dados}

Os instrumentos foram hospedados em uma página na Internet, cujo endereço de acesso foi encaminhado aos potenciais participantes, os quais foram recrutados por meio de redes sociais e listas de e-mails. Os dados obtidos foram analisados por meio de análise fatorial exploratória (AFE). Para esse fim, o tamanho da amostra foi considerado adequado, conforme critério de 100 casos e 10 casos por item do instrumento (Crocker \& Algina, 2009).

Após inspeção do banco de dados, nove casos foram excluídos por conterem respostas invariadas à ESQP, resultando em amostra de 213 casos. Não foram observados dados omissos. Os itens não apresentaram distribuição normal univariada e multivariada, conforme resultados dos testes de Kolmogorov-Smirnov 
e de curtose e assimetria multivariadas de Mardia. Uma vez que, a exceção de alguns tipos de extração (como a máxima verossimilhança), essas normalidades não são exigências em AFE (Pasquali, 2012; Tabachnick \& Fidell, 2009), os dados foram considerados adequados aos objetivos do estudo.

\section{Resultados}

Na matriz de correlações gerada para os nove itens, por meio de ACP, $72 \%$ das correlações foram iguais ou superiores a 0,30. Encontrou-se determinante de 0,046, cujo valor sugeriu ausência de multicolinearidade entre os itens (Field, 2013). Também foi encontrada medida de adequação da amostra de Kaiser-Meyer-Olkin (KMO) igual a 0,87. Esses achados indicaram que a matriz era fatorável. A análise paralela (Horn, 1965) e a análise visual do scree plot (Cattell,
1966) sugeriram a possibilidade de extração de até dois fatores.

$\mathrm{Na} A F E$, por fatoração dos eixos principais (avaliando a solução unifatorial do instrumento original), extraiu-se um fator, que explicou $37 \%$ da variância na matriz de dados. Todos os itens apresentaram cargas significativas (entre 0,47 e 0,71) e o fator também apresentou índice satisfatório de consistência interna (alfa de Cronbach igual a 0,84). Porém, restaram 69\% de resíduos não explicados superiores a 0,05 na matriz de correlações reproduzidas, indicando haver ainda variância a ser explicada na matriz.

Ao extrair dois fatores, por fatoração dos eixos principais com rotação oblíqua, os Fatores 1 e 2 explicaram $49 \%$ da variância na matriz de dados, restando $5 \%$ de resíduos na matriz de correlações reproduzidas. Os fatores se correlacionaram moderadamente $(r=0,47) \mathrm{e}$ apresentaram índices de consistência interna satisfatórios (Tabela 1).

Tabela 1. Descrição dos Itens ESQP e suas Cargas-fatoriais após Extração de Dois Fatores por Fatoração dos Eixos Principais com Rotação Oblíqua

\begin{tabular}{|c|c|c|c|c|}
\hline \multirow{2}{*}{ Itens } & \multirow{2}{*}{ Descrição } & \multicolumn{2}{|c|}{ Cargas-fatoriais } & \multirow{2}{*}{$h^{2}$} \\
\hline & & Fator 1 & Fator 2 & \\
\hline 8 & $\begin{array}{l}\text { Alguém com menos experiência profissional do que eu poderia desempenhar o meu cargo } \\
\text { tão bem quanto eu. }\end{array}$ & 0,90 & $-0,12$ & 0,73 \\
\hline 2 & A experiência profissional que eu tenho não é necessária para ter sucesso neste cargo. & 0,76 & $-0,04$ & 0,56 \\
\hline 5 & Meu treinamento anterior não está sendo totalmente utilizado neste cargo. & 0,65 & 0,16 & 0,55 \\
\hline 9 & Eu possuo mais capacidades do que eu preciso para desempenhar o meu cargo. & $-0,14$ & 0,76 & 0,49 \\
\hline 3 & Eu possuo habilidades profissionais que não são exigidas neste trabalho. & $-0,03$ & 0,67 & 0,43 \\
\hline 7 & Meu nível de educação formal é superior ao exigido pelo meu cargo. & 0,09 & 0,60 & 0,42 \\
\hline 1 & Alfa de Cronbach & 0,82 & 0,78 & \\
\hline
\end{tabular}

Nota. Cargas fatoriais significativas (acima de 0,30 ) escritas em negrito.

A presença de correlação significativa entre os fatores indicou a possibilidade de extração de fator de ordem superior (Thompson, 2004). Procedeu-se, então, à AFE da matriz de correlações dos Fatores 1 e 2, por fatoração dos eixos principais, sendo possível a extração de apenas um fator de segunda ordem, dada a presença de apenas dois fatores de primeira ordem. Ambos os fatores de primeira ordem apresentaram cargas fatoriais de 0,69 no fator de segunda ordem (Fator I), cuja consistência interna foi de 0,84 (alfa de Cronbach).

A fim de analisar a contribuição individual dos Fatores 1, 2 e I sobre as variáveis, bem como a variância 
explicada em cada nível (primeira ordem e segunda ordem), foi utilizada a solução de Schmid-Leiman (SSL; Schmid \& Leiman, 1957). A SSL é uma análise adicional à análise fatorial de ordem superior, que permite identificar as cargas e as variâncias da matriz de dados para cada fator, de primeira ordem e de ordem superior (Brown, 2015; Thompson, 2004). O fator de segunda ordem explicou $49 \%$ da variância explicada extraída, enquanto os fatores de primeira ordem explicaram $51 \%$ (Tabela 2).

Tabela 2. Cargas fatoriais e Comunalidades para os Itens nos Fatores de Primeira Ordem e no Fator de Segunda Ordem (Solução de Schmid-Leiman)

\begin{tabular}{|c|c|c|c|c|c|c|}
\hline \multirow{2}{*}{ Itens } & \multicolumn{3}{|c|}{ Cargas fatoriais } & \multicolumn{3}{|c|}{$h^{2}$} \\
\hline & Fator 1 & Fator 2 & Fator I & Fatores 1 e 2 & Fator I & Total \\
\hline 8 & 0,66 & $-0,09$ & 0,54 & 0,44 & 0,29 & 0,73 \\
\hline 2 & 0,56 & $-0,03$ & 0,49 & 0,31 & 0,24 & 0,55 \\
\hline 5 & 0,47 & 0,12 & 0,55 & 0,24 & 0,31 & 0,55 \\
\hline 4 & 0,39 & 0,11 & 0,47 & 0,17 & 0,22 & 0,39 \\
\hline 9 & $-0,10$ & 0,55 & 0,42 & 0,31 & 0,18 & 0,49 \\
\hline 3 & $-0,02$ & 0,49 & 0,44 & 0,24 & 0,19 & 0,43 \\
\hline 7 & 0,06 & 0,44 & 0,47 & 0,20 & 0,22 & 0,42 \\
\hline 6 & 0,19 & 0,40 & 0,55 & 0,19 & 0,30 & 0,49 \\
\hline 1 & 0,19 & 0,33 & 0,49 & 0,14 & 0,24 & 0,38 \\
\hline
\end{tabular}

Nota. Cargas fatoriais significativas (acima de 0,30 ) escritas em negrito

O Fator 1 foi constituído pelos Itens 2, 4, 5 e 8, que descreviam a percepção de possuir mais qualificações do que as necessárias para o cargo, sendo por isso denominado SQP-Necessidade. O Fator 2 foi constituído pelos Itens 1, 3, 6, 7 e 9, que descreviam a percepção de possuir mais qualificações que as exigidas para o cargo, sendo denominado SQP-Exigência. O Fator I, constituído pelos nove itens, foi denominado SQP-Global.

Os escores fatoriais da ESQP foram obtidos por meio da média aritmética das respostas aos itens, compreendendo os dois fatores de primeira ordem (escores SQP-Necessidade e SQP-Exigência) para o escore SQP-Global, quatro itens (Itens 2, 4, 5 e 8) para o escore SQP-Necessidade e cinco itens (Itens 1, 3, 6, 7 e 9) para o escore SQP-Exigência. Os escores da ESQP poderiam assumir valores entre 1,00 e 7,00.

A interpretação dos fatores sugeriu que as necessidades do cargo (as qualificações que os participantes percebiam serem utilizadas durante o exercício do cargo) difeririam das exigências (os requisitos formais para ocupar o cargo). Isso sugeriu que o fator SQP-Exigência estaria relacionado à sobrequalificação objetiva. Para testar essa relação, os participantes foram divididos em dois grupos, conforme a sobrequalificação objetiva. Como medida de sobrequalificação objetiva foi utilizada a sobre-educação, dada pela diferença entre o nível de escolaridade possuído pelo indivíduo e o nível de escolaridade exigido pelo cargo. Treze casos foram excluídos da análise por não possuírem escolaridade mínima. Assim, formaram-se dois grupos: sobre-educados (SE; $n=90$ ) e não sobre-educados (NSE; $n=110$ ).

$\mathrm{O}$ teste de Kolmogorov-Smirnov indicou que os escores violaram o pressuposto da normalidade. Por essa razão, a diferença entre os escores dos grupos foi testada por meio do teste não-paramétrico para medidas independentes de Mann-Whitney e foram reportadas as medianas dos escores (com respectivos erros-padrões e intervalos de confiança calculados por bootstrap). O Grupo SE apresentou escore SQP-Exigência (Mdn = 3,80; $E P=0,26$; IC95\%[3,20, 4,20]) maior que o do Grupo NSE (Mdn = 2,80; EP = 0,20; IC95\%[2,60, $3,40]$ ), com $U=6.211,00(p<0,01)$, embora com fraco tamanho de efeito $(r=0,16)$. Os escores SQP-Global e SQP-Necessidade não diferiram entre os grupos. Os escores SQP-Necessidade e SQP-Exigência também foram comparados entre si, para cada grupo, utilizando-se o teste não-paramétrico para medidas repetidas de Wilcoxon. Para o Grupo NSE, o escore SQP-Necessidade 
$(M d n=3,25 ; E P=0,26 ;$ IC95\%[3,00, 3,75]) foi maior que o escore SQP-Exigência ( $M d n=2,80 ; E P=0,20$; IC95\% $[2,60,3,40])$, $\operatorname{com} T=1.826,00(p<0,001 ; r=0,16)$. Não foi encontrada diferença significativa entre os escores para o Grupo SE.

\section{Discussão}

Os resultados da análise fatorial indicaram que a solução hierárquica, com dois fatores de primeira ordem e um de segunda, foi a mais adequada aos dados, quando comparada às soluções de um fator e de dois fatores. Isso decorreu do aumento significativo na variância explicada (de $37 \%$ para $49 \%$ ), na redução dos resíduos na matriz de correlações reproduzidas (de $69 \%$ para $5 \%$ ) e no acréscimo de um nível de análise (fator de segunda ordem). Essa estrutura fatorial diferiu do modelo adotado neste estudo (Edwards, 1991; Feldman, 1996; Kristof, 1996; Maynard et al., 2006) e não foi encontrada estrutura semelhante na literatura revisada.

Ao interpretar os fatores da ESQP, a análise dos itens do Fator 1 sugeriu que se referiam a possuir mais experiência profissional e educação formal do que as necessárias para o desempenho do cargo. Assim, o Fator 1 mensuraria a sobrequalificação percebida com base na necessidade de qualificação (educação formal e experiência profissional) para o cargo (SQP-Necessidade). Por sua vez, a análise dos itens do Fator 2 sugeriu que se referiam a possuir mais educação formal e habilidades do que as exigidas para o desempenho do cargo. Deste modo, o Fator 2 mensuraria a sobrequalificação percebida com base na exigência de qualificação (SQP-Exigência). O fator de segunda ordem (Fator I) foi interpretado como a sobrequalificação percebida (SQP-Global), isto é, a incongruência demandas-aptidões ou a incongruência entre a qualificação possuída pelo indivíduo e os requisitos do cargo. Este fator corresponderia ao fator único obtido pela SPOQ (Maynard et al., 2006).

A comparação entre os grupos encontrou o escore SQP-Exigência significativamente maior para o Grupo SE, não tendo sido encontrada diferença quanto ao escore SQP-Necessidade. Isso sugere que a sobrequalificação percebida se associou à objetiva, isto é, a sobrequalificação percebida foi maior na presença da sobrequalificação objetiva do que em sua ausência, como relatado por Zhang et al. (2016).

Ao comparar os escores SQP-Necessidade e SQP-Exigência, encontrou-se este significativamente menor que aquele, para o Grupo NSE. Isso sugere que a incongruência demandas necessárias-aptidões foi maior do que a incongruência demandas exigidas-aptidões. Em outras palavras, a diferença entre qualificação (aptidões) e necessidades do cargo foi maior do que a diferença entre qualificação e exigências para o cargo. Se a percepção do indivíduo acerca de sua qualificação for considerada uma constante (não variando segundo as demandas necessárias e as exigidas), pode-se inferir que, em média, os requisitos exigidos pelos cargos dos participantes não-sobrequalificados estavam acima das necessidades reais para seu exercício.

\section{Estudo 2}

O Estudo 2 foi realizado com os objetivos de: (1) testar e comparar três modelos estruturais para a ESQP (um fator, conforme o instrumento original; dois fatores; e hierárquico, com dois fatores de primeira ordem e um de segunda, conforme resultados do Estudo 1); e (2) testar a relação entre sobrequalificação percebida e atitudes no trabalho (comprometimento organizacional afetivo, satisfação no trabalho e intenção de saída), conforme realizado por Maynard et al. (2006) no desenvolvimento da SPOQ.

\section{Método}

\section{Participantes}

Participaram 293 trabalhadores (51\% mulheres), com média de idade de 37,73 anos ( $D P=10,85$ ). Trabalhavam em uma organização do setor de serviços, em média, havia 7,59 anos $(D P=8,91)$, em cargos que exigiam nível fundamental (19\%), médio (23\%), graduação (47\%) ou pós-graduação (6\%). Para $5 \%$ dos participantes não havia exigência de nível mínimo de escolaridade ou não souberam informar. Quanto ao nível de escolaridade, possuíam nível fundamental (7\%), médio (34\%), graduação (25\%) e pós-graduação (33\%). Três participantes (1\%) não informaram seu nível de escolaridade.

\section{Instrumentos}

Além da ESQP e de um questionário sociodemográfico, os participantes responderam a Escalas de: Comprometimento Organizacional Afetivo (ECOA), composta por 18 itens, respondidos em escala de cinco pontos ( 1 = nada; 5 = extremamente); Satisfação no Trabalho (EST) reduzida, composta por 15 itens, respondidos em escala de sete pontos ( $1=$ totalmente 
insatisfeito; 7 = totalmente satisfeito) e mensurando cinco aspectos da satisfação no trabalho (satisfação com colegas, chefia, salário, trabalho e promoções); e Intenção de Rotatividade (EIR), composta por três itens, respondidos em escala de cinco pontos ( 1 = nunca; 5 = sempre). Essas escalas foram desenvolvidas por Siqueira (1995).

\section{Procedimentos}

Os questionários foram entregues ao setor de recursos humanos, responsável por distribuí-los aos funcionários. Depois de preenchido pelo participante, o questionário era colocado em um envelope (que o acompanhava) e devolvido lacrado ao setor de recursos humanos, que os devolveria ao pesquisador. Foram distribuídos 382 questionários, dos quais 306 foram devolvidos (80\%). Treze destes estavam em branco ou com erros de preenchimento e foram excluídos, resultando em 293 questionários (77\%).

Os itens e os escores das escalas não apresentaram distribuição normal univariada (teste de Kolmogorov-Smirnov) e multivariada (teste de curtose e assimetria multivariadas de Mardia). Por essa razão foram utilizadas estatísticas não-paramétricas e análise fatorial confirmatória (AFC) com estimação por máxima verossimilhança robusta, utilizando o qui-quadrado Satorra-Bentler (SBX ${ }^{2}$ ) (Brown, 2015). Casos com dados omissos e respostas invariadas para cada escala foram excluídos, restando 265 casos para a ESQP, 285 para a ECOA, 281 para a EST e 123 para a EIR.

A AFC encontrou ajustamento bom para a EST $\left(\mathrm{SB \chi}{ }^{2}\right.$ relativo $=1,43 ; \mathrm{RMSEA}=0,04, \mathrm{IC} 90 \%[0,02,0,05]$; $\mathrm{CFI}=0,98$; alfa de Cronbach $=0,74$ a 0,92), perfeito para a EIR $\left(S B \chi^{2}=0,00\right.$; alfa de Cronbach $\left.=0,90\right)$ e pobre para a $\mathrm{ECOA}\left(\mathrm{SB} \chi^{2}\right.$ relativo $=3,70$; $\mathrm{RMSEA}=0,10$, IC90\%[0,09, 0,11]; CFI = 0,84; alfa de Cronbach = 0,94), sendo esta última medida excluída da análise. Os critérios foram $\mathrm{SB \chi}^{2}$ relativo $\left(\mathrm{SB} \chi^{2} / g l\right)$ menor ou igual a 2,00, RMSEA menor ou igual a 0,08 e CFI maior ou igual a 0,90 (Schumacker \& Lomax, 2010).

\section{Resultados}

O modelo de um fator apresentou: $\mathrm{SB} \chi^{2}$ relativo = 3,74; RMSEA = 0,10, IC90\%[0,08, 0,12]; CFI = 0,85; alfa de Cronbach $=0,80$. O modelo de dois fatores apresentou: $\mathrm{SB \chi} \chi^{2}$ relativo $=3,28$; $\mathrm{RMSEA}=0,09, \mathrm{IC} 90 \%[0,07$, $0,12]$; $\mathrm{CFI}=0,88$; alfa de Cronbach $=0,68$ e 0,71. $\mathrm{O}$ modelo hierárquico apresentou: $\mathrm{SB} \chi^{2}$ relativo $=3,08$; RMSEA = 0,09, IC90\%[0,07, 0,11]; CFI = 0,91; alfa de Cronbach $=0,80$, para o fator de segunda ordem (SQP-Global), 0,68 e 0,71, para os fatores de primeira ordem (SQP-Necessidade e SQP-Exigência).

Os participantes foram distribuídos a dois grupos, como no Estudo 1 (SE, com $n=117$; NSE, com $n=135$ ). O teste de Mann-Whitney indicou que o escore SQP-Exigência, do Grupo SE ( $M d n=3,60$; $E P=0,25 ;$ IC95\%[3,40, 4,20]), foi maior que o do Grupo NSE ( $M d n=3,40 ; E P=0,12 ;$ IC $95 \%[3,00$, $3,60]$ ), com $U=6.654,00$ ( $p<0,05 ; r=0,10)$. O escore SQP-Necessidade não diferiu entre os grupos. Para ambos os grupos o escore SQP-Exigência foi maior que o escore SQP-Necessidade (Grupo SE: $M d n=2,50$; $E P=0,34$; IC95\%[2,00, 3,25]; Grupo NSE: $M d n=2,25$; $E P=0,0,14 ;$ IC95\%[2,00, 2,50]), conforme resultados do teste de Wilcoxon, $T=5.470,00(p<0,001 ; r=0,29)$, para o Grupo SE, e $T=7.130,50(p<0,001 ; r=0,28)$, para o Grupo NSE.

Por meio do coeficiente de correlação de Spearman, foi testada a relação entre sobrequalificação percebida e atitudes no trabalho. O escore SQP-Global se correlacionou negativamente às satisfações com trabalho ( $\rho=-0,30 ; p<0,001 ; n=257$ ) e promoções ( $\rho=-0,24 ; p<0,001 ; n=257$ ). O escore SQP-Necessidade se correlacionou negativamente às satisfações com chefia ( $\rho=-0,14 ; p<0,05 ; n=257$ ), trabalho ( $\rho=-0,28 ; p<0,001 ; n=257$ ) e promoções ( $\rho=-0,15 ; p<0,05 ; n=257$ ). O escore SQP-Exigência se correlacionou negativamente às satisfações com trabalho ( $\rho=-0,24 ; p<0,001 ; n=257$ ) e promoções ( $\rho=-0,24 ; p<0,001 ; n=257$ ). Não foram encontradas correlações significativas entre sobrequalificação percebida e as demais variáveis mensuradas.

\section{Discussão}

Nenhum dos três modelos da ESQP testados por meio de AFC apresentou bons índices de ajustamento. Ainda assim, o modelo hierárquico mostrou melhores índices do que os modelos de um e dois fatores. A SQP-Exigência se associou à sobre-educação, tendo sido maior para os sobre-educados que para os não sobre-educados, mas não foi encontrada tal associação para a SQP-Necessidade. Adicionalmente, a SQP-Exigência foi maior do que a SQP-Necessidade, sugerindo que, em média, as exigências para os cargos dos participantes eram menores do que suas necessidades reais.

Dentre as relações observadas por Maynard et al. (2006) entre os escores da SPOQ e atitudes no trabalho, 
somente as correlações entre sobrequalificação percebida e as satisfações com chefia, trabalho e promoções foram encontradas neste estudo. No caso da ESQP, observou-se que, quanto maior a SQP-Necessidade, menores as satisfações com a chefia, o trabalho e as promoções, e quanto maior a SQP-Exigência, menores as satisfações com o trabalho e as promoções.

\section{Discussão geral}

A adoção da solução hierárquica permitiu uma visão mais ampla da estrutura da ESQP, acrescentando um nível de análise em comparação com a SPOQ. Apesar do modelo hierárquico, obtido no Estudo 1, não ter se ajustado aos dados do Estudo 2, ele foi o mais adequado em ambos os estudos. Raramente um modelo teórico se ajusta perfeitamente aos dados reais, de modo que a AFC busca encontrar um modelo que melhor se ajuste, dentre modelos alternativos (Schumacker \& Lomax, 2010).

A versão da SPOQ (Maynard et al., 2006), a ESQP, adaptada à língua portuguesa falada no Brasil, foi considerada adequada. Porém, diferiu teórica e estruturalmente do instrumento original, apresentando dois fatores não explicados pelo modelo teórico adotado (Edwards, 1991; Feldman, 1996; Kristof, 1996; Maynard et al., 2006). Essas diferenças devem ser consideradas com base em pelo menos dois pontos: o processo de adaptação e o procedimento de análise da ESQP.

Quanto ao processo de adaptação, considerou-se que o instrumento original apresentava validade de constructo, de modo que os procedimentos de operacionalização do constructo (Pasquali, 2010) foram substituídos pelos de tradução e retrotradução (Beaton et al., 2000; Borsa et al., 2012). Isso representou uma diferença metodológica significativa entre os estudos da SPOQ (Maynard et al., 2006) e da ESQP. Mas, essa diferença foi compreensível por, no caso da ESQP, se tratar de um estudo de adaptação. Assim, concluiu-se que a SPOQ e a ESQP mensuram o mesmo constructo, a sobrequalificação percebida.

Quanto aos procedimentos de análise, pode-se dizer que os da ESQP foram um pouco além daqueles realizados no desenvolvimento da SPOQ. A análise de validade da SPOQ foi realizada por meio de ACP tendo sido encontrado um único componente, para o qual os nove itens apresentaram cargas significativas (Maynard et al., 2006). Na verificação de evidências de validade da ESQP, além da ACP, por razões técnicas, decidiu-se por outros procedimentos. Após a ACP, a análise paralela indicou a existência de dois componentes e decidiu-se pela realização da análise fatorial com extração de dois fatores; a correlação entre os dois fatores extraídos indicou a presença de um fator de segunda ordem, o que levou à decisão por uma solução hierárquica.

Apesar das diferenças nos procedimentos de análise dos instrumentos original e adaptado, os nove itens da SPOQ (Maynard et al., 2006) foram retidos na ESQP, após análise empírica. Note-se ainda que, na ESQP, todos os itens apresentaram cargas significativas em ambos os níveis, de primeira e segunda ordem. Tanto na SPOQ quanto na ESQP, esses nove itens forneceram um escore, obtido por meio de média aritmética. $\mathrm{Na}$ ESQP, o escore fornecido pelos nove itens (escore SQP-Global) também representou a sobrequalificação percebida. A diferença residiu, então, nos escores dos fatores de primeira ordem (SQP-Necessidade e SQP-Exigência) presentes na ESQP. Esses fatores representam um nível mais específico, a partir da subdivisão da sobrequalificação percebida, com base nas necessidades e nas exigências do cargo. Conclui-se, então, que SPOQ (Maynard et al., 2006) e ESQP apresentariam uma medida comum, a sobrequalificação percebida, e que a ESQP forneceria duas medidas adicionais.

A diferença estrutural entre SPOQ (Maynard et al., 2006) e ESQP tem implicações teóricas e práticas para o estudo da sobrequalificação. Quanto à teoria, a ESQP seguiu o modelo da sobrequalificação (Edwards, 1991; Feldman, 1996; Kristof, 1996; Maynard et al., 2006) ao mensurar a sobrequalificação percebida (incongruência demandas-aptidões) por meio do fator SQP-Global. Contudo, os fatores SQP-Necessidade e SQP-Exigência não se ajustaram àquele modelo, segundo o qual as dimensões da sobrequalificação seriam educação formal, experiência profissional e habilidades.

Os fatores da ESQP foram constituídos por educação formal e experiência profissional, de um lado, e por educação formal e habilidades, de outro. Ao analisar os itens em cada fator, observou-se que um deles se referia a possuir mais educação formal e experiência do que as necessárias para o cargo e, o outro, a possuir mais educação formal e habilidades do que as exigidas pelo cargo. Isso sugeriu que a incongruência demandas-aptidões seria constituída por duas dimensões: incongruência demandas necessárias-aptidões; e incongruência demandas exigidas-aptidões.

Nos dois estudos aqui relatados, a SQP-Exigência se associou positivamente à sobre-educação, 
sendo que tal associação não foi observada para a SQP-Necessidade. Em outras palavras, a sobrequalificação percebida foi maior quando a sobre-educação (um indicador de sobrequalificação objetiva) esteve presente do que quando esteve ausente, o que é congruente com os achados de estudos anteriores (Maynard et al., 2015; Zhang et al., 2016). Antes da análise dos dados da ESQP, seria impossível supor as necessidades e exigências do cargo como dimensões da sobrequalificação percebida. É possível que o surgimento dessas dimensões tenha ocorrido devido a diferenças culturais nas crenças e valores com relação à percepção de sobrequalificação, entre as amostras do instrumento original (estadunidense) e a brasileira.

Como explicação a posteriori, a diferenciação entre demandas necessárias e exigidas pode ser suportada pelos estudos de Dejours (1997) e Green e Zhu (2010). O primeiro estudo diferenciou dois tipos de trabalho: o trabalho prescrito, que corresponderia às tarefas que o indivíduo deve realizar; e o trabalho real, que corresponderia às atividades que o indivíduo realiza. O trabalho prescrito poderia ser equiparado às demandas exigidas e, o trabalho real, às demandas necessárias. O segundo estudo classificou os requisitos para o cargo como reais ou formais, os quais também permitiriam supor a existência de demandas necessárias ou exigidas, respectivamente. Sugere-se que as incongruências demandas necessárias-aptidões e demandas exigidas-aptidões seriam dimensões pertencentes ao modelo da sobrequalificação, com base em Dejours (1997), Green e Zhu (2010) e nos resultados da análise da ESQP. Restaria saber se seriam dimensões da sobrequalificação ou da educação formal, experiência profissional e habilidades. Assim, os resultados da análise da ESQP descrevem a sobrequalificação percebida constituída por duas dimensões, agregando mais um nível à definição constitutiva desse constructo e à teoria da sobrequalificação.

Nos dois estudos, as SQP-Necessidade e SQP-Exigência diferiram significativamente entre si. No Estudo 1, para o grupo de não sobre-educados, a SQP-Necessidade foi maior que a SQP-Exigência. No Estudo 2, observou-se o inverso, a SQP-Exigência foi maior que a SQP-Necessidade, para ambos os grupos. A percepção do indivíduo acerca de sua qualificação provavelmente seria uma constante, independentemente de ele compará-la às necessidades ou às exigências de seu cargo; o que variaria seria sua percepção acerca das exigências para o cargo (baseada nos requisitos prescritos na descrição do cargo) e das necessidades do cargo (baseada em sua experiência, no exercício diário do cargo). Assim, infere-se que, no Estudo 1, as exigências para o cargo eram maiores que suas necessidades e, no Estudo 2, as necessidades do cargo superavam os requisitos para ele exigidos. Disso se sugere que a ESQP seria capaz de indicar a discrepância entre os requisitos exigidos para o cargo e suas reais necessidades.

As principais limitações deste estudo dizem respeito à amostra e a variáveis intervenientes. A amostra do Estudo 1 pode ser considerada pequena e não representativa quanto à região de origem, nível de escolaridade, setor, etc., prejudicando a generalização dos resultados. A amostra do Estudo 2 foi obtida em uma única organização e, portanto, possuindo menor variabilidade que a do Estudo 1.

Variáveis intervenientes provavelmente interferiram nos resultados. A região de origem dos participantes é um exemplo; a maioria dos participantes do Estudo 1 residia na região norte, menos industrializada e com baixos indicadores educacionais em comparação com a região sudeste. Também a adequação entre trabalho e formação dos participantes em ambos os estudos pode ter interferido nos resultados; não se sabe quais possuíam cargo compatível ou incompatível com sua formação, o que influencia a sobrequalificação. A profissão dos participantes seria outra variável interveniente; há mais opções de emprego para algumas profissões do que para outras, o que pode alterar as exigências de qualificação e, consequentemente, favorecer ou inibir a sobrequalificação. Em estudos futuros, com coleta de dados distinta da aqui relatada, será possível, e necessário, realizar nova análise fatorial (confirmatória ou até exploratória), a fim de testar a estrutura da ESQP.

\section{Considerações finais}

De modo geral, pode-se afirmar que o objetivo proposto neste estudo foi alcançado, tendo resultado em uma versão da SPOQ (Maynard et al., 2006) adaptada ao português brasileiro e adequada à mensuração e avaliação da sobrequalificação percebida. Mais estudos são necessários, a fim de testar a estrutura fatorial da ESQP, de modo a verificar se ela se mantém em outras amostras. Também seria necessário testar se a ESQP seria indicadora de sobrequalificação objetiva (por meio do fator SQP-Exigência) e de incongruência 
entre demandas necessárias e exigidas para o cargo, como sugerido neste estudo. Finalmente, estudos futuros poderiam analisar a relação entre sobrequalificação percebida, mensurada pela ESQP, e variáveis investigadas em estudos internacionais, a fim de verificar se seus resultados também ocorrem no Brasil.

\section{Referências}

Alfes, K., Shantz, A., \& Baalen, S. (2016). Reducing perceptions of overqualification and its impact on job satisfaction: The dual roles of interpersonal relationships at work. Human Resource Management Journal, 26(1), 84-101. doi: 10.1111/1748-8583.12094

Beaton, D.E., Bombardier, C., Guillemin, F., \& Ferraz, M. B. (2000). Guidelines for the process of cross-cultural adaptation of self-report measures. Spine, 25(4), 3186-3191. doi: 10.1097/00007632-200012150-00014

Borsa, J. C., Damásio, B. F., \& Bandeira, D. R. (2012). Adaptação e validação de instrumentos psicológicos entre culturas: algumas considerações. Paidéia, 22(53), 423-432. doi: 10.1590/S0103-863X2012000300014

Brown, T. A. (2015). Confirmatory factor analysis for applied research ( $2^{\mathrm{a}}$ ed.). Nova lorque: The Guilford Press.

Cattell, R. B. (1966). The scree test for the number of factors. Multivariate Behavioral Research, 1(2), 245-276. doi: 10.1207/ s15327906mbr0102_10

Cheng, B., Zhou, X., Guo, G., \& Yang, K. (2018). Perceived overqualification and cyberloafing: A moderated-mediation model based on equity theory. Journal of Business Ethics, 1-13. doi: 10.1007/ s10551-018-4026-8

Crocker, L., \& Algina, J. (2009). Classical and modern test theory. Mason: Cengage Learning.

Dejours, C. (1997). O fator humano. Rio de Janeiro: FGV.

Deng, H., Guan, Y., Wu, C.-H., Erdogan, B., Bauer, T., \& Yao, X. (2018). A relational model of perceived overqualification: The moderating role of interpersonal influence on social acceptance. Journal of Management, 44(8), 3288-3310. doi: 10.1177/0149206316668237

Edwards, J. R. (1991). Person-job fit: A conceptual integration, literature review, and methodological critique. In C. L. Cooper \& I.T. Robertson (Orgs.), International Review of Industrial and Organizational Psychology (Vol. 6, pp. 283-357). Chichester: John Wiley \& Sons.

Erdogan, B., Tomás, I., Valls, V., \& Gracia, F. J. (2018). Perceived overqualification, relative deprivation, and person-centric outcomes: The moderating role of career centrality. Journal of Vocational Behavior, 107, 233-245. doi: 10.1016/j.jvb.2018.05.003

Feldman, D. C. (1996). The nature, antecedents and consequences of underemployment. Journal of Management, 22(3), 385-407. doi: $10.1177 / 014920639602200302$

Field, A. (2013). Discovering statistic using IBM SPSS for windows (4 ed.). Londres: SAGE Publications.

Green, F., \& Zhu, Y. (2010). Overqualification, job dissatisfaction, and increasing dispersion in the returns to graduate education. Oxford Economic Papers, 62(4), 740-763. doi: 10.1093/oep/gpq002

Horn, J. L. (1965). A rationale and test for the number of factors in factor analysis. Psychometrica, 30(2), 179-185. doi: 10.1007/BF02289447

Hu, J., Erdogan, B., Bauer, T. N., Jiang, K., Liu, S., \& Li, Y. (2015). There are lots of big fish in this pond: The role of peer overqualification on task significance, perceived fit, and performance for overqualified employees. Journal of Applied Psychology, 100(4), 1228-1238. doi: 10.1037/apl0000008

Jackson, D., \& Collings, D. (2018). The influence of Work-Integrated Learning and paid work during studies on graduate employment and underemployment. Higher Education, 76, 403-425. doi: 10.1007/ s10734-017-0216-z

Johnson, W. R., Morrow, P. C., \& Johnson, G. J. (2002). An evaluation of a perceived overqualification scale across work settings. The Journal of Psychology, 136(4), 425-441. doi: 10.1080/00223980209604169

Kristof, A. L. (1996). Person-organization fit: An integrative review of its conceptualizations, measurement, and implications. Personnel Psychology, 49, 1-49. doi: 10.1111/j.1744-6570.1996.tb01790.x

Liu, S., Luksyte, A., Zhou, L., Shi, J., \& Wang, M. (2015). Overqualification and counterproductive work behaviors: Examining a moderated mediation model. Journal of Organizational Behavior, 36, 250-271. doi: 10.1002/job.1979

Lobene, E. V., \& Meade, A. W. (2013). The effects of career calling and perceived overqualification on work outcomes for primary and secondary school teachers. Journal of Career Development, 40(6), 508-530. doi: 10.1177/0894845313495512

Maciel, C. O., \& Camargo, C. (2013). Sobrequalificação no trabalho e sua influência sobre atitudes e comportamentos. Revista de Administração Contemporânea, 17(2), 218-238. doi: 10.1590/ S1415-65552013000200006

Maltarich, M. A., Reilly, G. \& Nyberg, A. J. (2011). Objective and subjective overqualification: Distinctions, relationships, and a place for each in the literature. Industrial and Organizational Psychology, 4(02), 236-239.

Maynard, D. C., Brondolo, E. M., Connelly, C. E., \& Sauer, C. E. (2015) I'm too good for this job: Narcissism's role in the experience of overqualification. Applied Psychology: An International Review, 64(1), 208-232. doi: 10.1111/apps.12031

Maynard, D. C, Joseph, T. A., \& Maynard, A. M. (2006). Underemployment, job attitudes, and turnover intentions. Journal of Organizational Behaviour, 27, 509-536. doi: 10.1002/job.389

Pasquali, L. (2010). Testes referentes a constructo: teoria e modelo de construção. In L. Pasquali (Org.), Instrumentação psicológica: fundamentos e práticas (pp. 166-198). Porto Alegre: Artmed.

Pasquali, L. (2012). Análise fatorial para pesquisadores. Brasília: LabPAM.

Schmid, J., \& Leiman, J. M. (1957). The development of hierarchical factor solutions. Psychometrika, 22(1), 53-61. doi: 10.1007/BF02289209

Schumacker, R. E., \& Lomax, R. G. (2010). A beginnes's guide to structural equation modeling ( $3^{\underline{a}}$ ed.). Nova lorque, NY: Routledge.

Siqueira, M. M. M.(1995). Antecedentes de comportamentos de cidadania organizacional: a análise de um modelo pós-cognitivo (Tese de doutorado não publicada). Universidade de Brasília, Brasília-DF.

Tabachnick, B. G., \& Fidell, L. S. (2009). Using multivariate statistics (6 ed.). Nova Jersey: Pearson Education.

Tebbe, E. A., Allan, B. A., \& Bell, H. L. (2019). Work and well-being in TGNC adults: The moderating effect of workplace protections. Journal of Counseling Psychology, 66(1), 1-13. doi: 10.1037/cou0000308

Thompson, B. (2004). Exploratory and confirmatory factor analysis: Understanding concepts and applications. Washington, DC: American Psychological Association.

Triana, M. C., Trzebiatowski, T., \& Byun, S.-Y. (2017). Lowering the threshold for feeling mistreated: Perceived overqualification moderates the effects of perceived age discrimination on job withdrawal and somatic

Estudos de Psicologia, 23(3), julho a setembro de 2018, 224-235 
symptoms. Human Resource Management, 56(6), 979-994. doi: 10.1002/hrm.21812

Wassermann, M., Fujishiro, K., \& Hoppe, A. (2017). The effect of perceived overqualification on job satisfaction and career satisfaction among immigrants: Does host national identity matter? International Journal of Intercultural Relations, 61, 77-87. doi: 10.1016/j.jijintrel.2017.09.001

Wassermann, M., \& Hoppe, A. (2019). Perceived overqualification and psychological well-being among immigrants. Journal of Personnel Psychology, 18(1), 34-45. doi: 10.1027/1866-5888/a000219

Weymer, A. S. Q., Maciel, C. O., \& Castor, B. V. J. (2014). A influência da sobrequalificação e da aprendizagem sobre a satisfação do indivíduo no trabalho. Revista Brasileira de Gestão de Negócios, 16(50), 96-109. doi: 10.1590/S1415-65552013000200006

Wu, C.-H., Tian, A. W., Luksyte, A., \& Spitzmueller, C. (2017). On the association between perceived overqualification and adaptive behavior. Personnel Review, 46(2), 339-354. doi: 10.1108/ PR-05-2015-0134

Zhang, M. J., Law, K. S., \& Lin, B. (2016). You think you are big fish in a small pond? Perceived overqualification, goal orientations, and proactivity at work. Journal of Organizational Behavior, 37, 61-84 doi: 10.1002/job.2024

João Moreira Gonçalves Neto, Mestre em Psicologia Social, do Trabalho e das Organizações, pela Universidade de Brasília (UnB). Endereço para correspondência: Travessa Eneas Pinheiro, 2626, Marco, Belém-PA. Telefone: (91) 3131-1911. E-mail: goncalvesnetojm@gmail.com

Jairo Eduardo Borges-Andrade, Ph.D. em Sistemas Instrucionais, pela Florida State University, Estágios de pós-doutorado realizados no International Food Policy Research Institute (1990); University of Sheffield e Rijksuniversiteit Gröningen (2001); Instituto Superior de Ciências do Trabalho e da Empresa (2010), é Docente Permanente do Programa de Pós-graduação em Psicologia Social, do Trabalho e das Organizações da Universidade de Brasília (UnB) e Professor Aposentado por esta mesma Universidade. E-mail: jairo.borges@gmail.com

Recebido em 30.Nov.16 Revisado em 25.Fev.19 Aceito em 22.Mar.19 